\title{
Poétiques de la marge et sur la marge dans les arts romanesques de Joseph Zobel et Conceição Evaristo
}

\author{
Vanessa Massoni Da Rocha \\ Universidade Federal Fluminense \\ vanessamassonirocha@gmail.com
}

Rebut: 1 de maig de 2018

Acceptat: 26 de juliol de 2018

RESUM

Poètiques del marge i sobre el marge en les arts novel-lístiques de Joseph Zobel i Conceiçao Evaristo

Aquest article està dedicat a estudiar el discurs sobre el marge en la tessitura novel-lesca del martiniquès Joseph Zobel i de la brasilera Conceição Evaristo. Es tracta d' analitzar les històries La Rue Cases-Negres (1950) i Banzo - records de la favela (2016) que parlen dels pobres de la perifèria i de les plantacions, éssers reduïts a l'anonimat de la dinàmica social de les ciutats.

Incorporant l'espai definit per Glissant com Neo-Amèrica (1996, p.15), el Brasil i Martinica comparteixen experiències colonials marcades pel comerç d'esclaus, la dicotomia entre els colonitzadors i els colonitzats i la formació de una jerarquia cultural, social, política i econòmica que allunya els negres i els blancs, els amos del poder i les víctimes que han patit qualsevol tipus d'explotació. A partir d'aquesta experiència, la presència de la marginalitat està molt arrelada en la societat.

PARAules ClaU

Joseph Zobel, Conceição Evaristo, post colonització, Poètica del marge.

RÉSUMÉ

Poétiques de la marge et sur la marge dans les arts romanesques de Joseph Zobet et Conceiçao Evaristo

Cet article se consacre à étudier le discours sur la marge dans la tessiture romanesque du martiniquais Joseph Zobel et de la brésilienne Conceição 
Evaristo. Il s'agit d'analyser les récits La Rue Cases-Nègres (1950) et Banzo - mémoires de la favela (2016) portant sur les pauvres de la périphérie et des plantations, êtres réduits à l'anonymat de la dynamique sociale des villes.

Intégrant l'espace défini par Glissant comme Neo-América (1996, p.15), le Brésil et la Martinique partagent les expériences coloniales marquées par la traite de l'esclavage, la dichotomie entre les colonisateurs et les colonisés et la formation d'une hiérarchie culturelle, sociale, politique et économique qui éloigne les Noirs et les Blancs, les détenteurs des pouvoirs et les victimes ayant subi toute sorte d'exploitation. De cette expérience, la présence de la marginalité s'avère très profondément présente dans la société.

MOTS-CLÉS

Joseph Zobel, Conceição Evaristo, (post)colonisation, Poétique de la marge.

\section{RESUMEN}

Poeticas novelescas del margen y sobre el margen en las artes novelescas de Joseph Zobel y Conceiçao Evaristo

Este artículo está dedicado a estudiar el discurso del margen en los libros del martinicano Joseph Zobel y la brasileña Conceição Evaristo. El objetivo es analizar las obras Rue Cases-Nègres (1950) y Banzo - memorias de la favela (2016) sobre los pobres de la periferia y las plantaciones, seres reducidos al anonimato de la dinámica social de las ciudades.

Integrando el espacio definido por Glissant como Neo-América (1996, p.15), el Brasil y la Martinica comparten las experiencias coloniales marcadas por la trata de esclavos, la dicotomía entre los colonizadores y los colonizados y la formación de una jerarquía cultural, social, política y económica que distancia a los negros y blancos, los que tienen el poder y las víctimas que han sufrido algún tipo de explotación. A partir de esta experiencia, la presencia de la marginalidad está muy presente en la sociedad.

Palabras Clave

Joseph Zobel, Conceição Evaristo, Post-colonización, Poética del margen.

\section{AbSTRact}

\section{Poetics of the Margin and about the Margin in the Novel Arts by Joseph} Zobel and Conceição Evaristo

This article is devoted to studying the speech on the margin in the romantic range of Martinique Joseph Zobel and Brazilian Conceição Evaristo. The aim is to analyze the stories Rue Cases-Nègres (1950) and Banzo - memoirs of the 
favela (2016) on the poor of the periphery and plantations, beings reduced to the anonymity of the social dynamics of cities.

Integrating the space defined by Glissant as Neo-América (1996, p.15), Brazil and Martinique share the colonial experiences marked by the slave trade, the dichotomy between the colonizers and the colonized and the formation of a cultural, social, political and economic hierarchy that distances blacks and whites, power holders and victims who have undergone any kind of exploitation. From this experience, the presence of marginality is very deeply present in society.

\section{KEYWORDS}

Joseph Zobel, Conceição Evaristo, (Post) colonization, Poetic of the margin.

Cet article rapproche les réalités postcoloniales du Brésil et de la Martinique, espaces ayant vécu l'entreprise coloniale - des Portugais et des Français, respectivement, la traite négrière et l'esclavage durant des siècles. À ce sujet, Edouard Glissant, intellectuel et écrivain martiniquais, insiste sur le dialogue historique, social et culturel entre ces territoires à partir du concept de Neo-America : «La Neo-America - que ce soit au Brésil, sur les côtes de la Caraïbes, dans les îles ou dans le sud des États-Unis fait l'expérience réelle de la créolisation à travers l'esclavage, l'oppression, la dépossession par les systèmes esclavagistes divers $»^{2}$. Ces expériences déclenchent des " séquelles » ${ }^{3}$, d'après le terme de l'abolitionniste français Victor Schœlcher, présentes de manière flagrante dans la vie des Noirs et des gens issus des communautés les plus défavorisées à nos jours. Schœlcher énumère, lors d'une allocution en 1880, le préjugé de couleur, les partialités de la justice et les inégalités de droits de représentation et d'instruction comme des héritages de la situation d'assujettissement imposée aux esclaves. Certes, le maintien des hiérarchies sociales et les difficultés d'ascension sociale des Noirs et des plus

\footnotetext{
${ }^{1}$ Joseph ZOBEL, La Rue Cases-Nègres, Présence Africaine, Paris, 2015, p. 272.

${ }^{2}$ Édouard GLISSANT, Introduction à une poétique du divers, Gallimard, Paris, 1996, p. 15.

${ }^{3}$ Victor SCHOELCHER, Esclavage et colonisation, Presses Universitaires de France, Paris, 2008, p. 101.
} 
modestes financièrement demeurent des prémisses de ces sociétés inégales organisées autour des privilèges des classes aisées.

Comparée à de nombreuses reprises à l'auteure américaine Toni Morrison, l'écrivaine brésilienne Conceição Evaristo déclare :

C'est flatteur. À vrai dire, mon grand désir est de la connaître. C'est vrai que l'on nous compare souvent, une de mes amies a même fait sa thèse sur ce sujet. Je pense que cela a un lien avec ce qu'Edouard Glissant appelait la 'transversalité historique', le fait que la traite des noirs ait laissé un héritage similaire à tous les peuples, quelle que soit leur destination ${ }^{4}$

Dans ce sens, l'Amérique revendique ses multiples appartenances et filiations : elle « est fille de l'Afrique ${ }^{5}$ » et fraternellement reliée par la mémoire historique qui la caractérise, fruit d'une « culture composite ${ }^{6} »$.

Christiane Taubira, ministre de la justice française et auteure de la proposition de loi visant reconnaître la traite négrière et l'esclavage comme crimes contre l'humanité en 2001, prêche que « c'est la responsabilité des pouvoirs publics de prendre acte des inégalités présentes enracinées dans l'injustice passée et de susciter dialogue, concertation, compromis et consensus pour les éradiquer ${ }^{7} »$. Toutefois, l'abolition de l'esclavage n'a pas assuré le développement de politiques publiques pour la réinsertion sociale des anciens esclaves et de leurs descendants, ce qui n'a pas réduit le clivage entre les Noirs et les Blancs, entre les aisés et les humbles et entre les héritiers des maitres et de ceux des esclaves. L'abolition de l'esclavage en Martinique date de 1848 tandis que le Brésil détient le honteux titre de dernier pays des Amériques à promouvoir la libération des captifs, en 1880. Cet épisode contribuera pleinement au racisme et à la disparité d'opportunités au sein de la société brésilienne.

Dans ce contexte, la présence d'une poétique de la marge et sur la marge dans la tessiture littéraire attire notre attention et met en évidence le refus des exclusions et de l'anonymat d'une parcelle significative de ces populations. En Martinique, Joseph Zobel publie en 1950 le chef d'œuvre

\footnotetext{
${ }^{4}$ Martin LAVIELLE, « Conceição Evaristo, la romancière noire qui bouscule le Brésil ». https:// bibliobs.nouvelobs.com/actualites/20170717.OBS2211/conceicao-evaristo-la-romanciere-noirequi-bouscule-le-bresil.html, 2017, s/p.

${ }^{5}$ Paulina CHIZIANE, O canto dos escravos, Matiko e Arte, Maputo, 2017, p. 88.

${ }^{6}$ Édouard GLISSANT, op. cit., p. 60.

${ }^{7}$ Christiane TAUBIRA, L'esclavage raconté à ma fille, Éditions Philippe Rey, Paris, 2015, p. 121.
} 
La Rue Cases-Nègres et au Brésil, Conceição Evaristo édite en 2006 Becos da Memória dont la traduction en langue française paraîtra en 2016 sous le titre de Banzo - mémoires de la favela. Les deux romans seront étudiés au long de cet article grâce à la manière poétique et poignante dont ils traitent les thématiques de la marginalisation, de la violence et de l'oppression subies par les plus démunis en Martinique et au Brésil. Dans les deux récits, il est question du regard d'un enfant qui transforme son écriture en témoignage et en lutte contre la domination et l'asservissement. Prêts à dénoncer la précarité de leurs routines et de leurs voisins, ces enfants mettent en relief les multiples pénuries et toutes sortes d'exclusions imposées aux êtres de la marge.

La Rue Cases-Nègres est le troisième roman de Zobel, paru chez Présence Africaine après Les Jours immobiles (1946) et Diab'-là (1947). Son premier roman a été censuré par les autorités. La Rue Cases-Nègres est écrit dans une époque de grande effervescence politique et culturelle aux Antilles, à savoir : la départementalisation de 1946, la publication du Discours sur le colonialisme et du Discours sur la négritude de Césaire, étapes fondamentales du mouvement de la Négritude de Césaire, Damas et Senghor et, finalement, la parution de Peau Noire, masques blancs de Frantz Fanon. À ce momentlà, Zobel habitait en France où il a suivi une formation en littérature, en art dramatique et en ethnologie à la Sorbonne, à Paris. Il était en même temps professeur adjoint au Lycée François 1er de Fontainebleau, ville où il a rédigé l'intégralité du roman. En 1957, Zobel déménage au Sénégal où il vit pendant une quinzaine d'années. C'est l'époque de la présidence de Léopold Sédar Senghor et Zobel se dédie à la vie intellectuelle du pays, en produisant d'émissions culturelles à la radio sénégalaise. Il était aussi peintre, dessinateur, aquarelliste, céramiste et maître en art floral japonais. Cet artiste polyvalent s'est éteint en France en 2006 à l'âge de 91 ans.

Françoise Vandenwouwer raconte les débuts littéraires de Zobel et la création d'un nom de plume:

La carrière littéraire de Joseph Zobel démarre aux Antilles, durant la seconde guerre mondiale, l'île est alors sous l'autorité du gouvernement de Vichy. Il publie des nouvelles et des articles dans le journal Le Sportif, 'hebdomadaire sportif, littéraire \& d'information'. Les gens qui écrivent dans cette publication ne sont pas journalistes, ce sont pour la plupart des fonctionnaires, instituteurs, gens de l'administration. Leurs articles sont soumis à la censure, ils sont 
contraints de prendre des pseudonymes. Mais tout le monde à Fort-de-France sait que celui qui signe Kay-Mac-Zo est Joseph Zobel ${ }^{8}$

La journaliste défend que «le roman de Zobel est une œuvre de résistance car l'écrivain parlait aux Martiniquais de leur vie, ils se reconnaissaient dans ses histoires. Il leur parlait de leur culture, de leur réalité sociale. Il leur révélait l'existence d'une identité créole ${ }^{9}$ », ce qui lui a valu le titre d' " acteur de la décolonisation ». Joseph Zobel a joué un rôle incontournable dans la " décolonisation de la connaissance ${ }^{10}{ }^{\text {» }}$ et de la pensée antillaises après la colonisation. Il a réussi à mettre en valeur l'imaginaire antillais au détriment des matrices françaises et européennes qui s'imposaient dans les anciennes colonies. Dans ce sens, il est précurseur du mouvement de la Créolité de Bernabé, Chamoiseau et Confiant dont les prémisses sont

comprendre ce qu'est l'Antillais. Percevoir ce que signifie cette civilisation caribéenne encore balbutiante et immobile. Avec Depestre, embrasser cette dimension américaine, notre espace au monde. A la suite de Frantz Fanon, explorer notre réel dans une perspective cathartique. Décomposer ce que nous sommes tout en purifiant ce que nous sommes par l'expose en plein soleil de la conscience des mécanismes cachés de notre aliénation. ${ }^{11}$

Née en 1946, Conceição Evaristo est une militante du mouvement noir brésilien, avec une intense participation dans les événements liés au militantisme politico-social. Sa mère était blanchisseuse et la famille habitait dans une favela dans la zone sud de la ville de Belo Horizonte, la capitale de Minas Gerais. Elle a travaillé comme domestique et institutrice avant de suivre des études universitaires et de se faire diplômer en Littérature Comparée dans un doctorat à l'Universidade Federal Fluminense en 2011. Il faut souligner le fait que Conceição Evaristo a été la seule de ses huit sœurs et frères à suivre des études universitaires. Ses premiers écrits ont été publiés en 1990 dans le recueil d'œuvres littéraires afro-brésiliennes Cadernos Negros mais le début de la rédaction de ses textes date de plus lontemps. Écrit en 1977/1978, Becos

\footnotetext{
8 Françoise VANDENWOUWER, Ecrivains, acteurs de la décolonisation. https://www. pointculture.be/article/focus/ecrivains-acteurs-de-la-decolonisation-ii-joseph-zobel/, 2015, s/p.

${ }^{9}$ Ibid., s/p.

${ }^{10}$ Grada KILOMBA, Descolonizando o conhecimento. In http://www.goethe.de/mmo/ priv/ 15259710-STANDARD.pdf, 2013, s/p.

11 Jean BERNABE, Patrick CHAMOISEAU \& Raphaël CONFIANT, Eloge de la créolité, Gallimard, Paris, 1993, p. 21-22.
} 
de memória a attendu presque trente ans pour être publié. Premier roman à être rédigé par Conceição Evaristo il a fait preuve de toutes les difficultés vécues par l'écrivaine : méfiance éditoriale avec des manuscrits rejetés ou ignorés et difficultés de prendre en charge les frais de publication d'un roman. Il ne paraîtra qu'en 2006, trois ans après Ponciá Vivêncio (L'Histoire de Poncia en édition française de 2015), ouvrage vendu à plus de 20.000 exemplaires. En 2015 son livre Olhos d'água a été lauréat du Prix Jabuti, le plus prestigieux du Brésil, dans la catégorie Contes et chroniques. Les livres de Conceição Evaristo connaissent des traductions en anglais, français, espagnol et italien. De nos jours, une campagne populaire pour que l'écrivaine soit admise à l'Académie Brésilienne de Lettres prend de plus en plus forme.

Evaristo raconte dans la préface de L'Histoire de Poncia comment elle a débuté dans le monde littéraire et la perception politique de sa condition de marginalisée :

À la fin de mon primaire, en 1958, j'ai reçu mon premier prix de littérature en gagnant un concours de rédaction qui avait pour intitulé : 'Pourquoi je suis fière d'être Brésilienne'. Sur la qualité de la rédaction, il y eut consensus ; sur l'attribution du prix, il y eut des divergences. J'étais loin d'être une élève exemplaire. On attendait d'une petite fille Noire et pauvre et de sa famille une certaine passivité. Ce n'était pas le cas. Les membres de ma famille avaient une conscience, même diffuse, de leur condition de Noirs, de pauvres et de favelados ${ }^{12}$.

Elle met en avant l'importance de la découverte de l'écriture littéraire: «La limitation de l'espace physique et la pauvreté économique dans laquelle nous vivions trouvaient une compensation dans une fiction innocente, unique moyen dont je disposais pour vivre mes rêves ${ }^{13}$ ». D'après Evaristo, l'acte d'écrire pouvait dédommager, en quelque sorte, l'exiguiité et la précarité de sa vie à la favela. Écrire, c'était une manière de s'offrir d'autres réalités et de surmonter, par l'imagination, la pénurie qui l'entourait. À partir de cette expérience, elle a pu comprendre toutes les potentialités de la fabulation. Dans ce contexte, Huston explique que « réel-réel : cela n'existe pas, pour les humains. Réel-fiction seulement, partout, toujours, dès lors que nous vivons dans le temps. La narrativité s'est développée en notre espèce comme technique

\footnotetext{
${ }^{12}$ Conceição EVARISTO, L'Histoire de Poncia, Anaconda, Paris, 2015, p. 9.

${ }^{13}$ Ibid., p. 12.
} 
de survie ${ }^{14} »$. À vrai dire, Evaristo a très vite compris que les fictions ne sont jamais innocentes, car elles se nourrissent de la liberté qu'elles n'arrêtent pas de propager. C'est pourquoi écrire de la marge et sur la marge s'avère un acte d'insubordination et de subversion pour faire face à la (sur)vie, pour la peupler de possibilités.

Zobel et Evaristo ne se sont jamais connus et ont publié dans des moments différents - lui dans les années 1950 en France et elle dans les années 2000 à Rio de Janeiro. Evaristo s'inscrit dans l'écriture féminine et noire au Brésil et ces conditions ont ralenti son accès à la publication, vu qu'elle était doublement marginalisée au sein de la société brésilienne. Elle commence à occuper la scène littéraire à l'âge de 60 ans bien qu'elle ait commencé à écrire et à publier bien avant. Les différences entre leurs trajectoires toutefois ne sont pas très grandes. Bien au contraire, les deux intègrent des familles très modestes dans lesquelles les femmes deviennent des poteaux-mitans, "le pilier de l'organisation sociale ${ }^{15} »$. Fils d'une employée de maison et d'un chauffeur de maître, Zobel a été élévé par la grand-mère maternelle à la plantation. Evaristo, quant à elle, n'a pas connu son père et habitait avec sa mère lavandière et ses frères dans une favela. En plus, les deux se dédient à l'enseignement travaillant à l'Éducation nationale. Certes, les deux écrivains ont connu une ascension sociale et économique grâce aux études et à leur persévérance dans le monde littéraire.

Les deux ont également vécu des migrations, thématiques incorporées dans leurs écrits. Zobel a structuré son roman La Rue Cases-Nègres autour de trois chapitres : le premier reproduit l'époque de son enfance à la plantation au Petit-Morne, le deuxième, son enfance-adolescence au Petit-Bourg et le troisième, sa vie à Fort-de-France, capitale de la Martinique. Son exode rural marque l'accomplissement de ses aspirations de fuir la misère des plantations à la campagne et d'affronter la capitale avec ses maisons en béton et ses institutions pour fréquenter l'école. À son tour, Tite-Maria, de Banzo mémoires de la favela, devient une conteuse d'histoires-écrivaine prête à garder les mémoires à elle et aux voisins de la favela. Son texte s'organise autour des récits fragmentés composant une mosaïque plurielle de vies anonymes oubliées par le pouvoir public. En ce qui concerne les migrations, elle se voit obligée par la défavélisation à vivre ailleurs, laissant derrière elle l'espace de sa naissance et de son enfance. D'après la narratrice, « il y avait la peur, l'incertitude, l'imprévisibilité du lendemain ; Mais il y avait aussi la ténacité,

\footnotetext{
${ }^{14}$ Nancy HUSTON, L'espèce fabulatrice, Actes Sud, Paris, 2008, p. 17.

${ }^{15}$ Daniel MAXIMIN, Les fruits du cyclone, Seuil, Paris, 2006, p. 46.
} 
la force, le désir de vie ${ }^{16} »$. Dans un espace marqué par l'analphabétisme, TiteMaria découvre dans le monde des lettres une manière de résister au risque d'effacement associé à la démolition de la favela. Elle accompagne plusieurs habitants, les écoute, leur pose des questions à guise de dresser un portrait d'un espace plein de vie condamné à la disparition. De cette manière, même si les casebres seront détruits et l'espace remodelé et offert aux spéculations immobilières, la mémoire de la favela, de ses ruelles, de ses fêtes et de ses modes de vie demeure préservée. À son tour, le narrateur José Hassan découvre que discuter avec des gens lui apporte « un détail nouveau, grâce auquel se rapproche et se précise, au jour au jour, le panorama de la Route $\operatorname{Didier}^{17} »$. Autant que Tite-Maria, José Hassan met l'accent sur les gens, leur vécu, leurs impressions, leurs expériences. Le duo contemple les récits mémoriels, la beauté résiliente des réminiscenses, les promesses du souvenir.

Le dialogue littéraire entre les ouvrages en question fait preuve d'un regard vers la force, la beauté et la résistance présentes dans les territoires de la marge. Zobel et Evaristo composent des romans très expressifs à propos des dilemmes quotidiens à être surmontés et nomment des personnages effacés dans la dynamique des grands centres. Il s'agit d'un procédé littéraire de mise en valeur des gens qui occupent les places les plus baisses de la hiérarchie sociale. En d'autres termes, c'est une tentative de subvertir les emplacements étanches au sein des sociétés tout en plaçant la marge au centre du regard, des discussions et des projecteurs. Il est question de rompre les barrières persistant à éloigner les gens des marges et de les mettres à l'écart de la société et de ses débats les plus urgents. Il s'agit également d'appliquer des efforts pour que la marge quitte les coulisses et l'invisibilité pour occuper la scène et son protagonisme pleinement. Et tout cela à travers ses arts de faire et de dire longtemps méconnues - voire pourchassées - du canon artistique, en général, et littéraire, en particulier.

Conceição Evaristo raconte les nombreux périples pour surmonter ses difficultés tout en rêvant d'une ascension sociale par le biais des études. Les humiliations, les injustices, la précarité et l'exploration du travail des enfants s'imposaient toujours comme des obstacles de force flagrante contre lesquels il ne fallait point succomber :

C'est à huit ans que j'obtins mon premier emploi domestique - le premier d'une longue série. Je travaillais chez mes patronnes. J'accompagnais mes frères,

\footnotetext{
${ }^{16}$ Conceição EVARISTO, Banzo - mémoires de la favela, Anaconda, Paris, 2016, p. 208.

${ }^{17}$ Joseph ZOBEl, op. cit.,p. 261.
} 
mes sœurs et les enfants des voisins à l'école. J'aidais tout ce petit monde aux devoirs - empochant par la même occasion quelques sous. Je prêtais main forte à ma mère et à ma tante ; j'allais chercher les paquets de linge sale, je lavais le tout, et ramenais les balluchons chez les patronnes. J'ai même échangé avec certains professeurs des heures de tâches ménagères contre des cours particuliers, leur attention à l'école et surtout des livres - toujours didactiques pour moi, mes sœurs et mes frères. Gagner un peu d'argent avec les restes des riches que nous ramassions dans les poubelles fut aussi un mode de survie que nous avons expérimenté ${ }^{18}$.

Dans les entrecroisements entre biographie et fiction, la déclaration d'Evaristo rencontre des échos dans les exploitations imposées au personnage José Hassan de La Rue Cases-Nègres. Madame Léonce décide d'accueillir le petit et de lui donner un espace à manger pour qu'il ne refasse pas le long trajet école-maison au moment du déjeuner. L'aide et l'hospitalité de Mme Léonce ne tardent pas à devenir des cauchemars. D'abord, le petit se voit empêché d'entrer chez sa « protectrice » :

la seule chose qui ne m'enchante pas, c'est d'aller déjeuner dans le corridor de Mme Léonce. J'en souffre peut-être dans mon amour propre et, en outre, l'intérieur de cette maison dont je n'ai jamais vu que le corridor et une pièce sombre où j'entre à peine le matin pour déposer mon petit sac, m'inspire je ne sais quelle répulsion à cause du peu d'attention qu'on m'y accorde. J'ai la nette impression que dans le couloir je suis considéré comme étant dehors tout à fait $^{19}$.

Ensuite, le gamin reçoit l'autorisation pour accéder à la cuisine et commence à rendre des services obligatoires à la femme. Il fait la vaisselle, cire des chaussures, fait des commissions, balaie la cours et finit par rentrer toujours en retard en classe à cause des tâches multiples qu'il doit accomplir. Il avoue : «j'avais toujours peur de Mme Léonce que, sans le vouloir, je détestais à cause de ce sentiment de rabaissement dont je ne cessais de souffrir chez elle $^{20}$ ». Sa colère augmente et il vocifère à lui-même : « combien de temps allait durer cette torture $?^{21} »$, « combien de malheurs encore allait me porter

\footnotetext{
${ }^{18}$ EVARISTO, Conceição. « Conceição Evaristo par Conceição Evaristo » in L'Histoire de Poncia, Anaconda, Paris, 2015, p. 7.

${ }^{19}$ Joseph ZOBEl, op. cit., p. 110.

${ }^{20}$ Ibid., p. 119.

${ }^{21}$ Ibid., p. 123.
} 
cette détestable femme ?22 » avant de fuguer et ne plus jamais revenir chez elle. Il apprend que la bonté et la solidarité envers les Noirs peuvent cacher des motivations secrètes liées à la manutention des privilèges des classes les plus aisées et de l'utilisation à leur gré de la force de travail des plus dépourvus, y compris des enfants.

En ce qui concerne les femmes domestiques, Hassan mentionne « la circonspection, le conformisme, l'obséquiosité qui marquaient l'attitude et les moindres propos des domestiques - les seules personnes avec qui j'avais contact - en disaient assez sur la soumission qu'ils vouaient à leurs maîtres et leur respect pour ce lieu qu'ils s'appliquaient à ne troubler aucunement, se méfiant de leur exubérance de nègres, et s'astreignant à vivre aussi effacés que possible ${ }^{23}$ ». Il réitère : « je ne m'accoutumais pas à cette indigence passive des domestiques ${ }^{24}$ » et comparait leur métier au larbinisme: « il me semblait que le temps ne passait pas assez vite pour m' amener au jour où $\mathrm{j}$ 'allais travailler afin de délivrer ma mère, et ma grand-mère surtout, de la servilitude ${ }^{25} »$. Il rêvait de libérer ses figures maternelles de l'oppression du travail très mal rémunéré des Noirs, travail qui sacrifiait la santé des femmes et les poussait vers la maladie, la chute et la mort. Exception à la « sélection naturelle » d'une société injuste, il comprend qu'il a «bifurqué26 ${ }^{26}$ en n'ayant pas suivi la destinée des natifs de la rue Cases-Nègres. Il a réussi à briser un cercle vicieux qui s'applique pour que les marges se réduisent au territoire de la marge et ne prospèrent jamais. En d'autres mots, il a rompu la dynamique 'naturelle' d'exclusion, de délimitation de ses possibilités et de confinement des uns pour le maintien des privilèges des autres. Lui, fils d'une domestique et petit-fils d'une employée de plantations a échappé d'un engrenage social qui lui était fort défavorable pour gagner les capitales, les écoles, le monde des réjouissances.

Le 'bifurquement' de José Hassan est également vécu par Tite-Maria :

Maman Joana avait appris toute seule, en observant attentivement les lettres, pendant ses heures libres dans les maisons où elle travaillait. Cela expliquait peut-être son grand désir et l'effort qu'elle avait fait pour que ses enfants apprennent à lire. (...) Tite-Maria apprenait puis enseignait ce qu'elle apprenait

\footnotetext{
${ }^{22}$ Ibid., p. 124.

${ }^{23}$ Ibid., p. 253.

${ }^{24}$ Ibid., p. 255.

${ }^{25}$ Ibid., p. 271.

${ }^{26}$ Ibid., p. 257.
} 
à ses petits frères et aux enfants du voisinage. Tite-Maria grandissait, lisait, grandissait $^{27}$.

L'écrivaine Evaristo affirme que "C'est dans un contexte scolaire marqué par des pratiques pédagogiques tantôt excellentes, tantôt exécrables que je découvris avec plus d'intensité notre condition de Noir et de pauvre ${ }^{28} »$. Si l'école est fortement valorisée dans les romans, la faim et les difficultés pour se maintenir dans l'enseignement méritent aussi l'attention des narrateurs. Tite-Maria avoue que «souvent, la faim les accompagnait sur le chemin, car les quelques pièces du pain servaient à acheter un cahier, un crayon, une gomme. Les enfants marchaient vite et attendaient impatiemment le goûter distribué à la récréation ${ }^{29}$ ». José Hassan décrit à son tour la faim ressentie : « la faim, malgré mes efforts pour y rester sourd, pour la tromper, en faisant le plein d'eau à la fontaine, me poussa à déambuler dans le Haut-Morne, et dans ces parages, tous les midis, une aubaine m'échéait, comme par enchantement ${ }^{30} »$.

Les deux romans font allusion à la précarité alimentaire et à la difficulté de se procurer du sucre. Tite-Maria évoque la privation de gâteau : « les gamins demandaient toujours à Custódia de faire un gâteau. Mais il n'y avait pas assez de sucre, il y en avait à peine pour le café dilué et le biberon du petit ${ }^{31}$ » tandis que José Hassan décrit une chasse à trésor chez lui. Le petit et sa bande voulaient manger du sucre mais n'en trouvaient pas chez eux : «Ma m'man a pas de boîte à sucre, dit Gesner. C'est le dimanche seulement qu'elle achète deux sous de sucre pour faire du cafée $e^{32} »$. Hassan décide d'emmener les amis chez lui et ils commencent à chercher la boîte à sucre, que M'mam Tine, la grand-mère de José, cache de la gourmandise du petit-fils à cause de la vie austère qu'ils mènent. Étant donné que l'acquisition de sucre est coûteuse, elle finit par en faire un usage très limité. "Le sucre se trouve dans une boîte de fer-blanc ; mais dénicher cette boîte, voilà l'affaire ! M'man Tine a le génie de trouver des cachettes et d'y escamoter sa boîte à sucre sans que je m'en aperçoive ${ }^{33}{ }^{\prime}$. Cet épisode finit avec un bol cassé, par le désespoir des petits qui craignent l'incontournable punition.

\footnotetext{
${ }^{27}$ Conceição EVARISTO, op. cit., 2016, p. 70.

${ }^{28}$ Conceição EVARISTO, op. cit., 2015, p. 8.

${ }^{29}$ Conceição EVARISTO, op. cit., 2016, p. 70.

${ }^{30}$ Joseph ZOBEl, op. cit., p. 129.

${ }^{31}$ Conceição EVARISTO, op. cit., 2016, p. 93.

${ }^{32}$ Joseph ZOBEl, op. cit.,p. 30.

${ }^{33}$ Ibid., p. 29.
} 
Il faut remarquer que la consommation de sucre dans l'espace des plantation des canne à sucre en Martinique devient une aventure et une confirmation des privilèges des uns et des privations des autres. La grandmère n'arrive pas à avoir dans son dépotoir le produit final des cannes qu'elle entretien et coupe de ses propres mains. Le fruit de son travail ne lui est point accessible. Sa diète alimentaire fait preuve des inégalités sociales à toutes les domaines. Fatiguée, M'man Tine fait un discours à José pour le faire comprendre les problèmes de sa vie : «Tes maladies, c'est pour moi. Tes crises de vers, c'est pour moi. Et te laver, t'essuyer, t'habiller ! Pendant que toute la journée tu inventes toutes sortes de tracas pour moi, comme si j'en avais pas assez de mes coups de soleil, des averses, des coups de tonnerre, et de la houe avec laquelle il me faut gratter la terre coriace du béké34 ». Le Béké est le blanc créole propriétaire des plantations aux Antilles, descendant des premiers colons français à la Caraïbe.

Issus des communautés très pauvres, la vie de résilience des écrivains Zobel et Evaristo s'imbrique aux intrigues développées dans les romans privilégiés, d'indéniables contours autobiographiques. Zobel raconte les péripéties de José Hassan de l'enfance dans les plantations à la vie universitaire à Fort-de-France. À son tour, Evaristo raconte la vie de plusieurs habitants d'une favela à Minas Gerais en voie d'être démolie à travers le regard de Tite-Maria. Les deux récits mettent en scène les enjeux de la subalternité, la condition de la négritude, l'héritage colonial de l'esclavage dans le concept " favela cases-nègres ${ }^{35}$ », l'école, la pénurie alimentaire et les intempéries accompagnant les personnages.

Dans un jeu littéraire indéniable, Joseph Zobel nomme son personnage de José et Conceição Evaristo choisit son prénom, Maria, pour Tite-Maria. Le prénom de l'écrivaine est composé : Maria da Conceição. Les auteurs ont attribué à leurs protagonistes des prénoms très populaires - et bibliques - des leurs pays, ce qui confère un caractère plus collectif aux drames vécus par leurs personnages. On pourrait classifier l'entreprise littéraire de Zobel et Evaristo comme des autofictions, d'après les définitions de Vincent Colonna :

Par complaisance, manque d'imagination ou par une impérieuse nécessité intérieure, l'écrivain utilise ainsi sa biographie comme matière, pour une forme narrative où il s'abrite derrière un personnage romanesque. Et pour que cette attitude narrative soit conduite jusqu'à son terme, il est nécessaire que l'écrivain laisse entendre que son texte est une confession, qu'il encourage une lecture

\footnotetext{
${ }^{34}$ Ibid., p. 44.

${ }^{35}$ Conceição EVARISTO, op. cit., 2016, p. 82.
} 
en partie référentielle, comme Goethe avec Werther. Le roman personnel n'est donc qu'à demi-fictif, son contenu et l'effet qu'il recherche sont aussi autobiographiques ${ }^{36}$.

Face à une vie austère, Hassan et Tite-Maria deviennent des conteurs d'histoires et racontent leurs parcours et celui de leur entourage grâce à la puissance de la mémoire, de l'oralité et de l'écriture. C'est la revanche de la résistance pour surmonter l'amnésie de la chronique sociale et revendiquer le protagonisme longtemps effacé des Noirs appauvris et victimes de plusieurs exclusions. Il s'agit d'un mouvement littéraire de mise en valeur de la marge et d'une critique des jeux de force du capitalisme. Evaristo imbrique les domaines de la fiction et de sa vie personnelle quand elle explique sa tragique expérience de défavélisation : « J'ai achevé l'École Normale dans l'état de Minas Gerais en 1971. Période particulièrement diffcile pour ma famille et beaucoup d'autres qui subissaient de plein fouet le plan de 'défavélisation' de la ville. Ils nous envoyaient vivre en périphérie. En nous éloignant du centre de Belo Horizonte, nous ne gagnions rien, à part une plus grande pauvreté ${ }^{37}$ ».

L'auteure définit son roman Banzo - mémoires de la favela et son procédé littéraire : «J'écris. Je témoigne. Un témoignage où les images se mêlent, un moi-maintenant tirant un moi-petite fille dans les rues de Belo Horizonte. Et comme l'écriture et le vécu se (con)fondent, je poursuis cet écrit-vécu ${ }^{38}$ ». Ce mélange entre écriture et vie devient très explicite dans le terme forgé par Conceição Evaristo pour expliquer sa production : « l'écritvie »; en portugais, Escrevivência. Le concept fait référence aux interfaces entre souvenir personnel et mémoire collective, entre le présent des Noirs et de leurs ancêtres et s'appuie sur les rapports entre le vu, le vécu et l'écriture. Il prétend réhabiliter en quelque sorte les textes qui peuvent être appréhendés comme de 'simples' témoignages, ce qui finit par les circonscrire dans une catégorie moins raffinée, moins prestigieuse, voire sans qualité littéraire. Il faut remarquer, dans ce sens, que le travail pionnier d'écriture des marges effectué par Carolina Maria de Jésus, écrivaine afro-brésilienne des favelas qui a encouragé toutes les générations postérieures, est souvent mis en question à cause de son langage non normatif. La valeur littéraire de ses ouvrages, dont Le dépotoir émerge comme le chef d'œuvre, a besoin d'être à plusieurs reprises

\footnotetext{
${ }^{36}$ Vincent COLONNA, L'autofiction, essai sur la fictionalisation de soi en littérature, https://tel. archives-ouvertes.fr/tel-00006609/document, 1989, p. 9.

${ }^{37}$ Conceição EVARISTO, op. cit., 2015, p. 10.

${ }^{38}$ Ibid., p. 14.
} 
confirmée par des académiciens/lecteurs/critiques devant les attaques subies par des groupes plus conservateurs.

Zobel et Evaristo, il est important de le souligner, ne tombent pas dans les pièges de textes exotiques et pittoresques plein de victimisations que l'on pourrait naïvement associer aux écrivains qui privilégient la thématique de la marge. Leurs fictions méritent des accueils chaleureux du public lecteur et des institutions académiques, en plus de la consécration des prix spécialisés. Leurs textes occupent incontestablement les programmes des cours universitaires et des études à plusieurs niveaux. Evaristo s'avère en ce moment l'écrivaine noire la plus en évidence au Brésil et a représenté la littérature brésilienne lors du Salon du livre de Paris en 2018. Zobel, à son tour, a une œuvre largement reconnue parmi laquelle La Rue Cases-Nègres demeure le symbole. Le roman intègre la liste des lectures de formation obligatoires aux Antilles. Porté à l'écran par la martiniquaise Euzhan Palcy en 1982, avec la collaboration de François Truffaut et de l'auteur lui-même, le long métrage remporte dix-sept prix internationaux dont le Lion d'Argent en1983 et le César de la meilleure première œuvre en 1984.

À guise de conclusion, parler de la marge et l'accueillir au sein de leurs romans sont des mots d'ordre dans l'intégralité des écrits de Zobel et Evaristo. D'un côté, les deux s'inspirent d'une réalité qui leur est fort connue et, de l'autre, ils envisagent dans leurs productions une manière de combler les vides de représentation qui accompagnent tantôt les Noirs tantôt les plus fragilisés de la structure sociale. Dans le roman de Zobel, le narrateur José Hassan avoue : « rien ne m'a jamais paru aussi bien conçu pour dégoûter de toute étude, de toute lecture même, que ces brochures intitulées : Le Cid, Le Misanthrope, Athalie ${ }^{39}$ ». Ses lectures préférées étaient des « ouvrages hors $\mathrm{du}$ programme et relatifs à la vie des nègres ${ }^{40} »$. Hassan explique : «ces livres (...) me passionnaient plus profondément que tous ces récits de la vie des rois numérotés, leurs guerres et leur mort, que j'apprenais et oubliais continuellement ${ }^{41}{ }^{»}$. Il ressentait un « vibrant orgueil ${ }^{42}$ » de cette image miroitée de soi-même que les romans évoquaient.

Il est certain que " la misère ne tue pas de mort violente ${ }^{43}$ », phrase mise en exergue de cette étude. Elle tue de manière lente et cruelle et elle

\footnotetext{
${ }^{39}$ Joseph ZOBEl, op. cit., p. 268.

${ }^{40}$ Ibid., p. 293.

${ }^{41}$ Ibid., p. 293.

${ }^{42}$ Ibid., p. 293.

${ }^{43}$ Ibid., p. 272.
} 
s'inscrit dans une dynamique de manque, d'exclusion et de détresse. Les productions littératures de la marge, dont Joseph et Conceição Evaristo font partie, décident de mettre à lumière cette misère dans toutes ses répercussions et prolongements. Ils la mettent à nu pour exposer le monde des êtres assujettis, pour briser l'anonymat et la non-information. Certes, il est question de renverser la dynamique sociale, chercher un nouveau paradigme et d'anéantir la misère, l'abandon et l'exploration des êtres démunis pour qu'ils ne meurent plus dans l'ombre, l'oubli et l'indifférence.

\section{Références bibliographiques}

Jean BERNABE, Patrick CHAMOISEAU \& Raphaël CONFIANT, Eloge de la créolité, Gallimard, Paris, 1993.

Vincent COLONNA, L'autofiction, essai sur la fictionalisation de soi en littérature, https://tel.archives-ouvertes.fr/tel-00006609/document, 1989.

Paulina CHIZIANE, O canto dos escravos, Matiko e Arte, Maputo, 2017.

Conceição EVARISTO, L'Histoire de Poncia, Anaconda, Paris, 2015.

EVARISTO, Conceição. «Conceição Evaristo par Conceição Evaristo » in L'Histoire de Poncia. Anaconda, Paris, 2015.

Conceição EVARISTO, Banzo - mémoires de la favela, Anaconda, Paris, 2016.

Édouard GLISSANT, Introduction à une poétique du divers, Gallimard, Paris, 1996.

Nancy HUSTON, L'espèce fabulatrice, Actes Sud, Paris, 2008.

Carolina Maria de JESUS, Le dépotoir, Stock, Paris, 1960.

Grada KILOMBA, Descolonizando o conhecimento. In http://www.goethe.de/ mmo/ priv/ 15259710-STANDARD.pdf, 2013.

Martin LAVIELLE, « Conceição Evaristo, la romancière noire qui bouscule le Brésil ». https://bibliobs.nouvelobs.com/actualites/20170717.OBS2211/ conceicao-evaristo-la-romanciere-noire-qui-bouscule-le-bresil.html, 2017.

Daniel MAXIMIN, Les fruits du cyclone, Seuil, Paris, 2006.

Victor SCHOELCHER, Esclavage et colonisation, Presses Universitaires de France, Paris, 2008.

Christiane TAUBIRA, L'esclavage raconté à ma fille, Éditions Philippe Rey, Paris, 2015.

Françoise VANDENWOUWER, Ecrivains, acteurs de la décolonisation. https://www.pointculture.be/article/focus/ecrivains-acteurs-de-ladecolonisation-ii-joseph-zobel/, 2015.

Joseph ZOBEL, La Rue Cases-Nègres, Présence Africaine, Paris, 2015. 